\title{
A novel detection method of spray droplet distribution based on LIDARs
}

\author{
Zheng Yongjun ${ }^{1}$, Yang Shenghui ${ }^{1}$, Yubin Lan ${ }^{2}$, Clint Hoffmann ${ }^{3}$, \\ Zhao Chunjiang ${ }^{4}$, Chen Liping ${ }^{5}$, Liu Xingxing ${ }^{1}$, Tan Yu ${ }^{1 *}$ \\ (1. College of Engineering, China Agricultural University, Beijing 100083, China; 2. College of Engineering, South China Agricultural \\ University/National Center for International Collaboration Research on Precision Agricultural Aviation Pesticides Spraying Technology \\ (NPAAC), Guangzhou 510642, China; 3. USDA ARS, College Station, TX 77845, USA; 4. National Engineering Research Centre of \\ Information Technology in Agriculture (NERCITA), Beijing 100097, China; 5. National Engineering Research Centre of Intelligent \\ Equipment for Agriculture (NERCIEA), Beijing 100080, China)
}

\begin{abstract}
During the process of plant protection in agriculture, the distribution and deposition of droplets or fog fields could directly influence the effectiveness and efficiency of spray. The traditional method of measurement of the distribution of droplets mainly used water sensitive papers, glass containers or flour to collect data and inverse results, while a new method of measurement based on the principle of reflection of LIDAR was presented. Droplets were the major targets of the study, and four important algorithms were primarily developed, including the recognition and extraction of targets, the superposition in time-domain, the calculation of effective ranges of distribution, and the development of 3D distribution models. Combined with these algorithms, in order to eliminate the environmental noise, the methods of Fuzzy Environment Matching and Secondary Filter were created and utilized. Meanwhile, the statistics was used for analysis of the duration of scanning as well as computation of the distribution, with enough datasets but the minimum length of time. The results of the experiments showed that the relative error of measurement was less than $7 \%$ and Relative Standard Deviation was less than 16\%, compared with the values of manual measurement. Furthermore, the 3D models were accurate and clarified in the wind-tunnel experiment. The completed system based on this method could adapt to the requirements of both indoor and outdoor detection. Besides, it is capable of the quantized detection of droplet distribution, providing an effective way of tests for spray technique, especially for the research of the application of plant protection by UAVs.
\end{abstract}

Keywords: droplets, distribution, detection, agricultural aviation, UAV

DOI: $10.25165 /$ j.ijabe. 20171004.3118

Citation: Zheng Y J, Yang S H, Lan Y B, Hoffmann C, Zhao C J, Chen L P, et al. A novel detection method of spray droplet distribution based on LIDARs. Int J Agric \& Biol Eng, 2017; 10(4): 54-65.

\section{Introduction}

Spraying pesticide is one of the most significant

Received date: 2016-01-29 Accepted date: 2016-11-22

Biography: Zheng Yongjun, $\mathrm{PhD}$, Associate Professor, research interests: intelligent detection for agriculture machine, Email: zyj@cau.edu.cn; Yang Shenghui, Master, research interests: computer measurement and control technology, Email: yang_sheng_h@126.com; Yubin Lan, PhD, Professor, research interests: precision agricultural aviation application, Email: ylan@scau.edu.cn; Clint Hoffmann, PhD, Research Leader, research interests: agricultural aviation application, Email: Clint.hoffmann@ars.usda.gov; Zhao Chunjiang, PhD, Research approaches of plant protection during the process of prevention of the disease and pest. In order to avoid the drawbacks of the traditional methods like high labour intensity and low efficiency, the technologies of

Scholar, research interests: agriculture information technology, Email: zhaocj@nercita.org.cn; Chen Liping, PhD, Research Scholar, research interests: intelligent agriculture equipment, Email: chenlp@nercita.org.cn; Liu Xingxing, PhD, Lecturer, research interests: intelligent detection for agriculture machine, Email: liuxingxing56285@163.com.

*Corresponding authors: Tan $\mathbf{Y u}, \mathrm{PhD}$, Professor, research interests: intelligent detection and control. Tel: +86-10-62736385, Email: tanyu@cau.edu.cn. 
agricultural aviation represented by the UAV have been gradually developed. However, the distribution of droplets or fog fields could influence the effectiveness and efficiency of the operation directly. Therefore, it is important to detect the range of spray of UAVs as well as study the laws of deposition and distribution of droplets.

At present, there are a lot of research about the laws of deposition and distribution of droplets. One of the most popular techniques is to lay the water sensitive paper, flour, or glassware to collect droplets. This method was used in previous research ${ }^{[1-4]}$ to specify the superior combination of parameters of UAVs during operation, such as the velocity of operation, height of operation affected by wind speed. Zheng et al. ${ }^{[5]}$ used this method to study the models between the prior parameters of operation of JF01-10 and penetration of droplets. Some researchers ${ }^{[6-9]}$ also applied this method to study the sizes, distribution and deposition of droplets from the ground-spray equipment. They focused on the effect caused by different conditions when operating, like height of spray, angles of nozzles and shapes of plants. Another popular technique is to re-collect the tracer by test tubes and then invert the situation of distribution by calculation of the tracer content. Guan ${ }^{[10]}$ and Qiu et al. ${ }^{[11]}$ used tracer like coccinellin to analyse the concentrations of droplets. It was based on the spectrophotometry method to measure the absorbance and then change into mass concentration to detect the quality of deposition. Foqué and Nuyttens ${ }^{[12]}$ used element chelate compound to analyse the influence of auxiliary air and angles of spray on the distribution of droplets. It was found that the auxiliary air had a great effect on the penetration of droplets. It is suitable for assessment of the regularities of distribution, but there are still some limitations, such as high costs and complex operation in both setting and post processing. Meanwhile, the collected data maybe damaged before analysis, resulting in inaccurate measurement.

High-speed video camera combined with image processing became a hotspot of application. Qi et al. ${ }^{[13]}$ used image processing to measure the sizes of droplets. The camera was used to catch the droplets collected by water sensitive papers, and the sizes were calculated by proper computer programs. Lu et al. ${ }^{[14]}$ used this kind of method, combined with PIDA, to measure some characteristics of droplets, such as sizes, perimeters, equivalent diameters, roundness and positions. It was also applied for redrawing the two-dimensional images of charged particles in electrostatic spray fields and tracking droplet marks in different frames. The method was exact and fast. However, Salyani and Fox ${ }^{[15]}$ pointed out that the size of image region, the quantity of droplets and the area of droplets had a significant effect on measurement. In other words, the obtained results of distribution could be influenced by the position and resolution of the image.

Using Laser Particle Size Analyser (LPSA) is also an effective way to detect droplets. Zhang et al. ${ }^{[16]}$ used the open wind-tunnel system and the Sympatec LPSA to test the sizes and quantities of droplets in different pressures, wind speeds and distances between the nozzle and the equipment. They found the relations among the factors above, the volumes of droplets and the levels of fog spectrum. Wedding et al. ${ }^{[17]}$ used LPSA to study the characteristics of air nozzles in wind tunnel. Two kinds of nozzles were set in a high-speed wind tunnel and different wind speeds were given to establish the fog spectrum. Sidahmed and Yates ${ }^{[18]}$ mainly concerned about the influence of probe size calibration on the measurement of typical agricultural spray with forwarding scattering spectrometer. Even though the results for different tests mentioned above showed high accuracy, LPSA could only analyze the distribution of droplets in $2 \mathrm{D}$ condition and might be limited by the environment of application.

CFD model was gradually concentrated due to its intuition and accuracy. Endalew et al. ${ }^{[19]}$ used the method of computer simulation to establish the $3 \mathrm{D}$ models of fruit trees. The influence of wind speed and direction on the air current of sprayers was analyzed, combined with the model. Even if the regularities among the jet velocity, the cross-wind velocity, and the wind direction were found, the results should be verified by experiments.

Currently, some scholars exploited sensors to detect droplets. Crowe et al. ${ }^{[20]}$ designed a sensor to detect the 
deposition of droplets. The forms of signals of droplets were discrete and digital, and the sensor could detect the signal when droplets fully located on the surface of circuit array. However, the sensor was effective for detection of rough spray, so the details need to be improved.

Laser measurement was widely applied in agriculture because of its higher accuracy. Different medium has different reflection of laser, so the existence of solid objects can be detected and measured in the scanning area based on the principle of laser-flight in time-domain. The common application of laser in agriculture is to scan out contours of objects and locate targets, such as measuring the heights of crops or fruit trees, scanning the shapes of grape vines, detecting the edge of crops or developing the $3 \mathrm{D}$ models ${ }^{[21-26]}$. In these applications, the signals caused by droplets or fog were regarded as the interference and filtered by laser sensors when operating.

In this paper, a novel method of measurement of the distribution of droplets was proposed with applying LIDARs as the major device of detection, in order to overcome the difficulties such as the recognition and extraction of droplets, the calculation of effective ranges of distribution and the development of 3D distribution models. Based on the method, the detection of droplets, the spatial scale characteristics of target data were extracted and the mathematic models were developed. The technique provides a kind of effective and technical means for research and application of agricultural aviation as well as the facilities of spray.

\section{Materials and methods}

\subsection{Materials and equipment}

The LIDAR, LMS511-20100, produced by SICK, was utilized as the major instrument of measurement. A detection system of the distribution of droplets was developed, combined with hardware devices, like the computer.

As shown in Figure 1, the LIDAR detects and measures the droplets; the data were then transmitted to the computer through the Ethernet, and recorded and stored by the computer. After that, the data were analyszd and processed and the results were drawn automatically. The characteristics of the LIDAR was listed in Table 1.

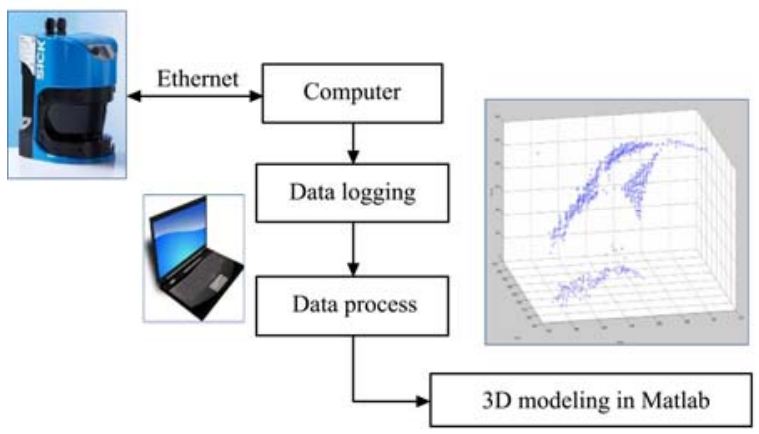

Figure 1 Hardware design

Table 1 Main technical parameters of the LIDAR LMS511-20100

\begin{tabular}{cc}
\hline Type & LMS511-20100 \\
\hline Scope of work & $0.7-80 \mathrm{~m}$ \\
The maximum magnitude with & $26 \mathrm{~m}$ \\
$10 \%$ reflectivity & \\
Scanning angle & Max $190^{\circ}$, adjusted in the parameter settings \\
Angle resolution & $0.167^{\circ}, 0.25^{\circ}, 0.333^{\circ}, 0.5^{\circ}, 0.667^{\circ}, 1^{\circ}$ \\
Scanning frequency & $25 / 35 / 50 / 75 / 100 \mathrm{~Hz}$ \\
Response time & $10-40 \mathrm{~ms}$ \\
Measurement accuracy & $\pm 12 \mathrm{~mm}$ \\
Spot size & $4.7 \mathrm{mrad}(4.7 \mathrm{~mm} / \mathrm{m})$ \\
Echoes & 5 \\
Laser wavelength & Infrared $(905 \mathrm{~nm})$ \\
Data interface & $\mathrm{RS} 232 / \mathrm{RS} 422 / \mathrm{Ethernet} / \mathrm{USB}$ \\
\hline
\end{tabular}

\subsection{Study of detection method of droplet distribution}

The detection method of droplet distribution is as shown in Figure 2.

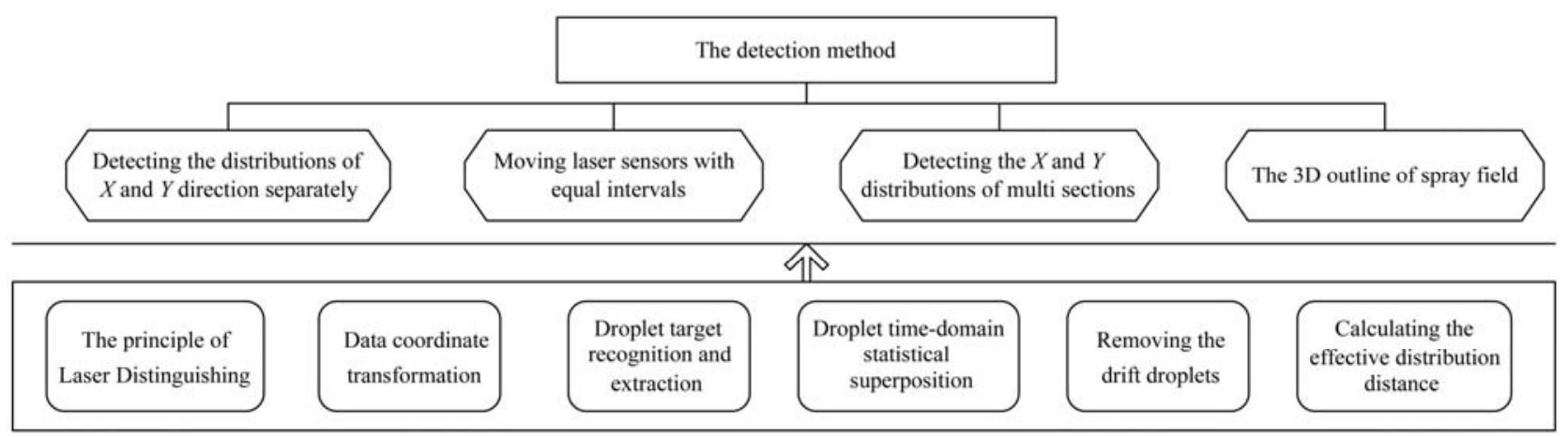

Figure 2 Technological theory of droplets distribution detection 
In order to detect the distribution of droplets in a certain direction within the two-dimensional section, the principle of laser distinguishing, the transformation of coordinates of data, the recognition and extraction of target-droplets, the statistical superposition of droplets in time-domain, the removing of drift droplets and the calculation of the effective range of distribution were designed. The distribution of droplets of $X$ - or $Y$-direction in a section could be measured by the method.

\subsubsection{Principle of laser distinguishing}

The principle of 5-echo technology was used to distinguish different objects ${ }^{[27]}$. It means that different objects have different reflection rates of laser. Thus, it could recognize many substances, such as glass, fog and dust, to deal with the problems like dynamic changes of target objects, bad weather conditions and low visibility,

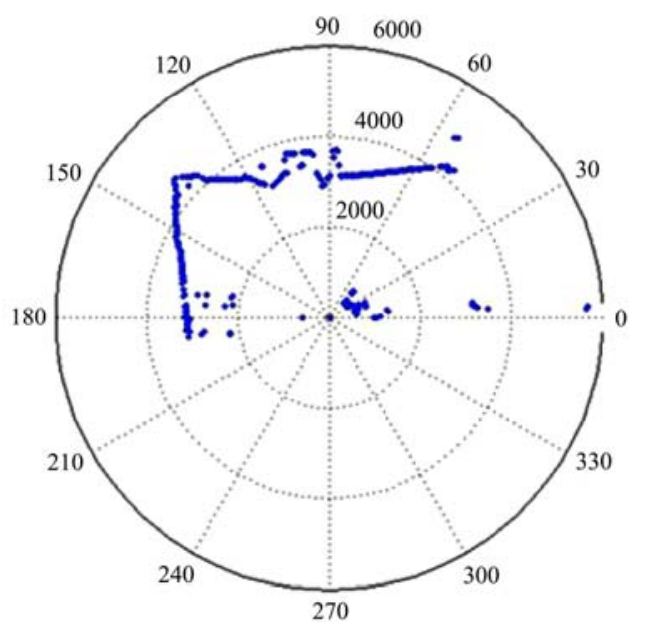

a. Polar coordinate so that the accuracy of the results of measurement could be ensured.

\subsubsection{Transformation of coordinates of data}

There are lot of datasets sent to the computer in polar format, including the distance $D$ and relative angle $\theta$. The key aspect of the study is to measure the distribution of droplets in a specific direction ( $X$-direction or $Y$-direction), so the data should be changed from the polar coordinate (Figure 3a) to the rectangular coordinate (Figure 3b). The Equations are as below:

$$
D_{x}=D \cdot \cos \theta, D_{y}=\mathrm{D} \cdot \sin \theta
$$

where, $D_{x}$ is the abscissa of the rectangular coordinate; $D_{y}$ is the ordinate of the rectangular coordinate; $D$ is the distance between the sensor and the object (the polar radius); $\theta$ is the relative angle in the polar coordinate (the polar angle).

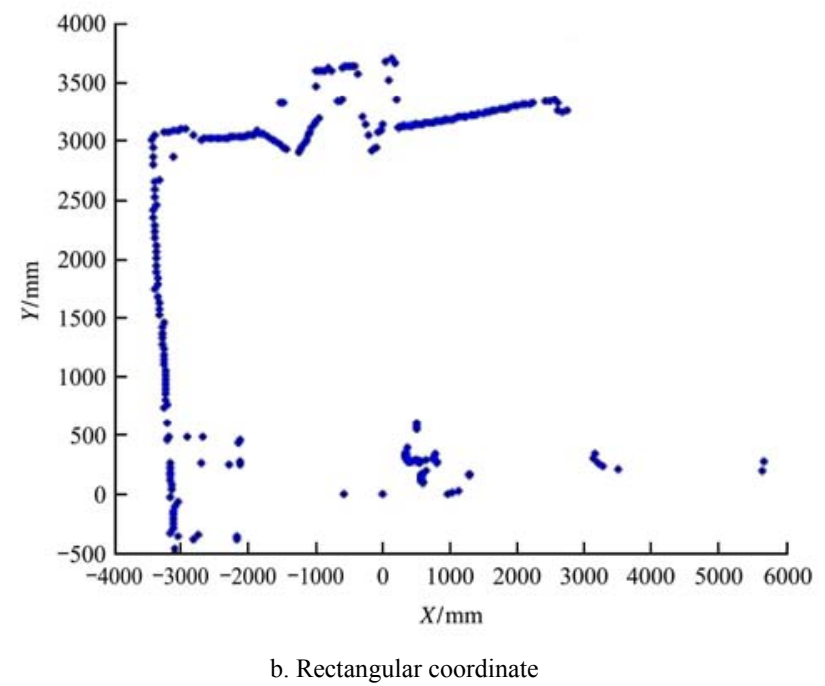

Figure 3 Two different coordinates

\subsubsection{Recognition and extraction of target-droplets}

The method of superposition to eliminate the environmental noise was studied, which can extract the target-droplets. Firstly, the LIDAR was used to collect the Environmental Data (ED). Then, the spray device was started and the LIDAR was used again to collect the Mixed Data (MD) including ED as well as the Droplet Data (DD). Finally, these two sets of data were superimposed to eliminate the ED to extract the DD.

As a result of the effect of some factors, like temperature drift or zero drift, however, there are some errors of data. If ED and MD were superimposed point by point simply and directly, the ED could not be eliminated thoroughly and it might affect the results.
Hence, the methods of Fuzzy Environment Matching (FEM) and Secondary Filter (SF) were developed to extract the DD.

The method of FEM was firstly used to eliminate the environmental noise. As shown in Figure 4, each of ED is used as a datum centre, and around this centre, the $X$-axis and the $Y$-axis are enlarged into a small matrix. Each of MD is compared with the matrix. If anyone of them falls into the ranges of the matrixes, this point may be affected by the environment and should be abandoned, otherwise, it should be kept and saved.

After that, Second Filter was applied to eliminate the left noise-point. Combined with the distance between the LIDAR and targets, the filtration intervals of $X$ and $Y$ 
directions were set separately. The data excluding the interval of any direction were eliminated so that the noise- points could be removed thoroughly. Figure 5 shows the MD (Figure 5a) and extracted DD (Figure 5b).
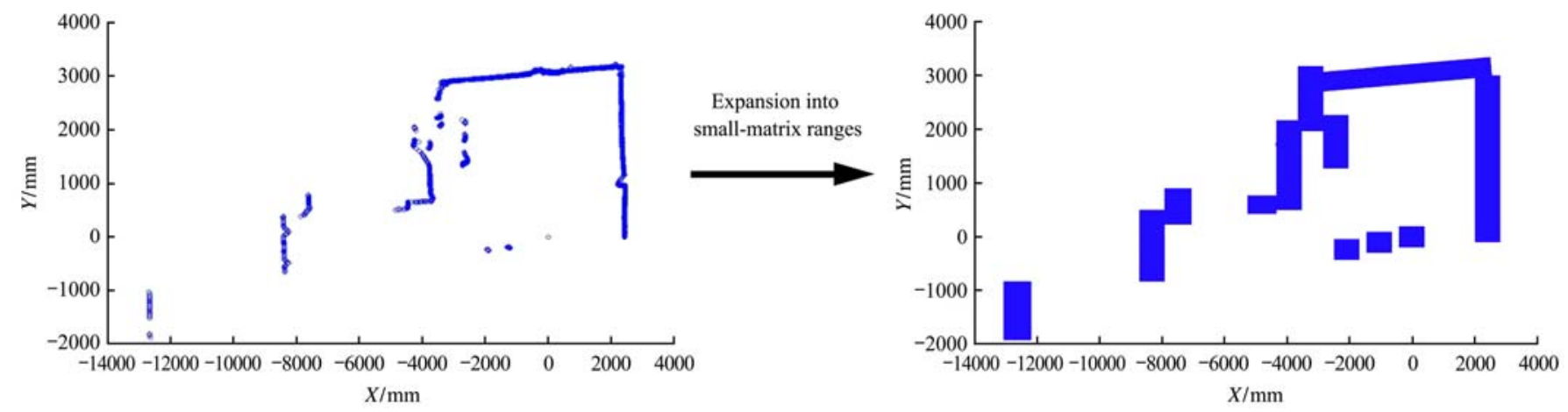

Figure 4 Method of Fuzzy Environment Matching (FEM)

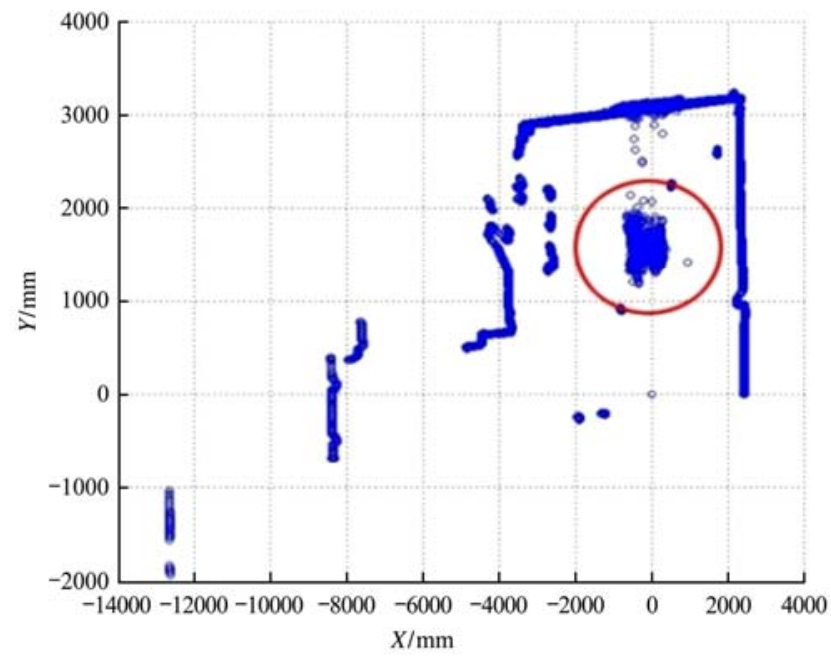

a. Mix-data

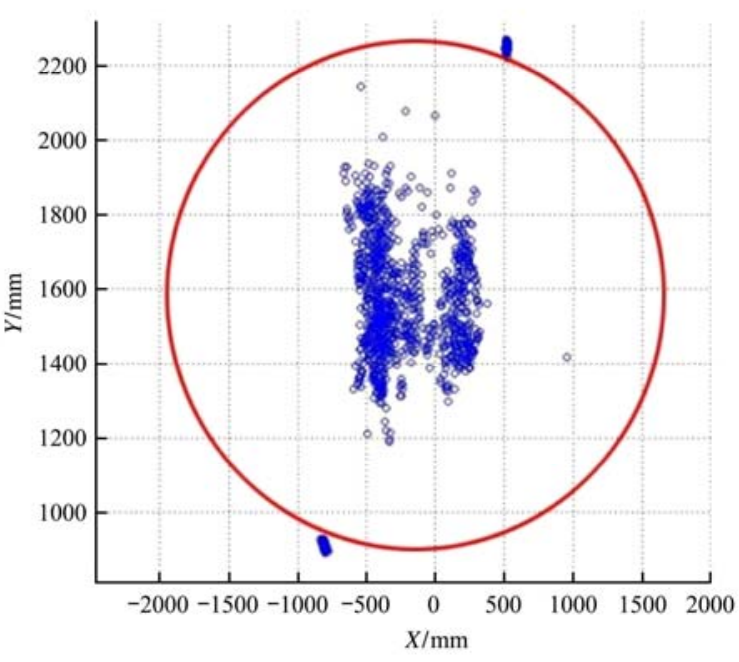

b. Extracted droplet-data

Figure 5 MD and extracted DD

\subsubsection{Statistical superposition of droplets in time-domain}

In order to analyze the impact from the duration of scanning on data sufficiency, a test was carried out, with a $12 \mathrm{~s}$ period of scanning. The result of the distribution of droplets in one scanning cycle is shown in Figure 6.

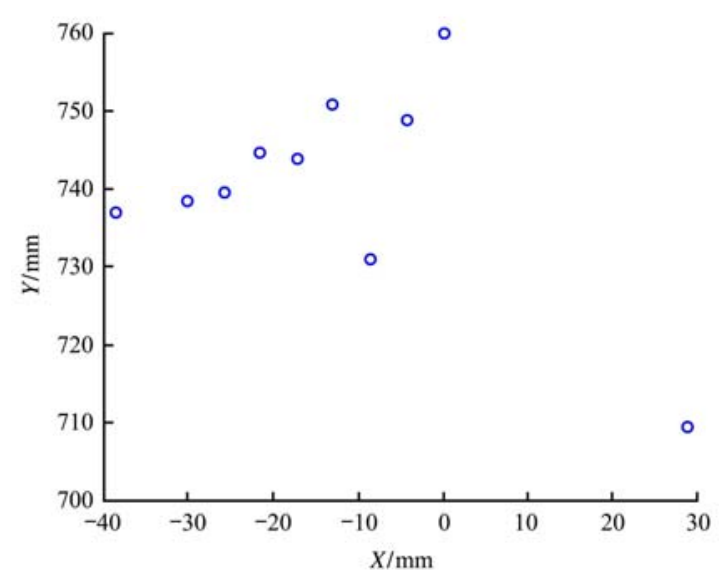

Figure 6 Extracted data of one scanning cycle
It shows that the overall distribution of droplets could not be indicated by one scanning cycle only. Therefore, the data of first four seconds, first five seconds, first seven seconds and first nine seconds were analyzed, shown from Figure 7a to Figure 7d, respectively.

The values of both left and right sides in the transverse distributions of droplets were changed into absolute values and then drawn as a line chart by EXCEL, as shown in Figure 8.

Meanwhile, the quantities of samples of $4 \mathrm{~s}, 5 \mathrm{~s}, 7 \mathrm{~s}$ and $9 \mathrm{~s}$ were also drawn as a line chart in Figure 9 by EXCEL.

From Figure 7 to Figure 9, it is known that the ranges of the distribution of droplets were expanding, and the quantities of samples had the same trend from $4 \mathrm{~s}$ to $7 \mathrm{~s}$. However, although the quantities of samples were expanding in $9 \mathrm{~s}$, the ranges of distribution were generally 
steady compared with $7 \mathrm{~s}$. Thus, for one process of scanning, the suitable period is about $8 \mathrm{~s}$, which could not

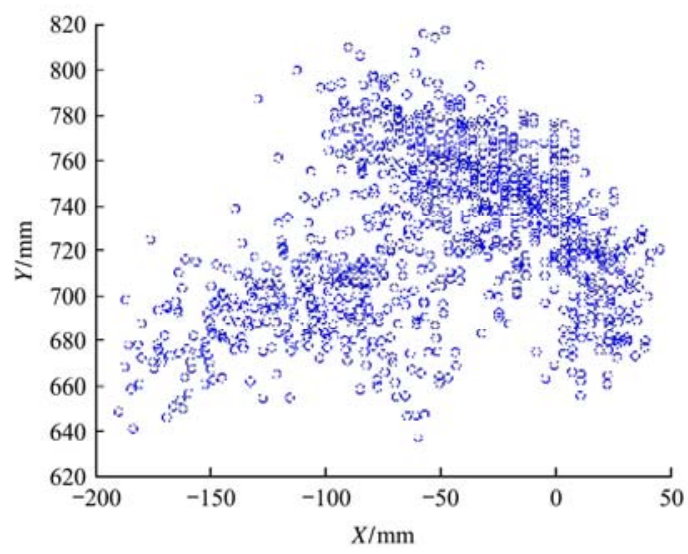

a. Scanning time with $4 \mathrm{~s}$

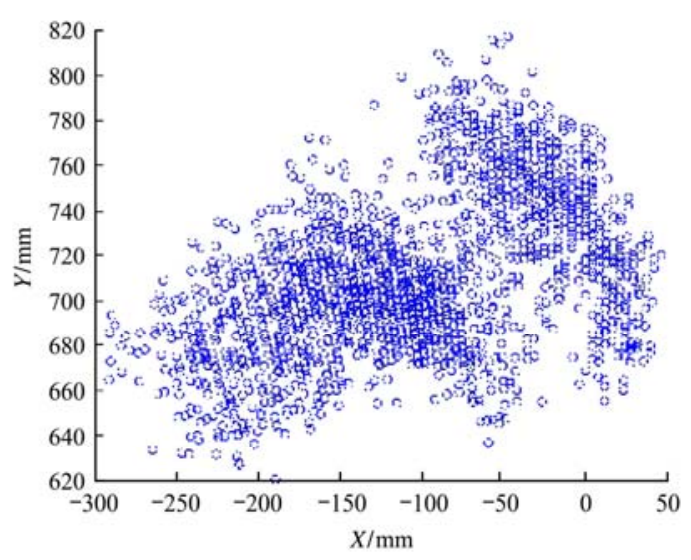

c. Scanning time with $7 \mathrm{~s}$ only denote the entire distribution but also reduce the quantities of sampling to increase the efficiency.

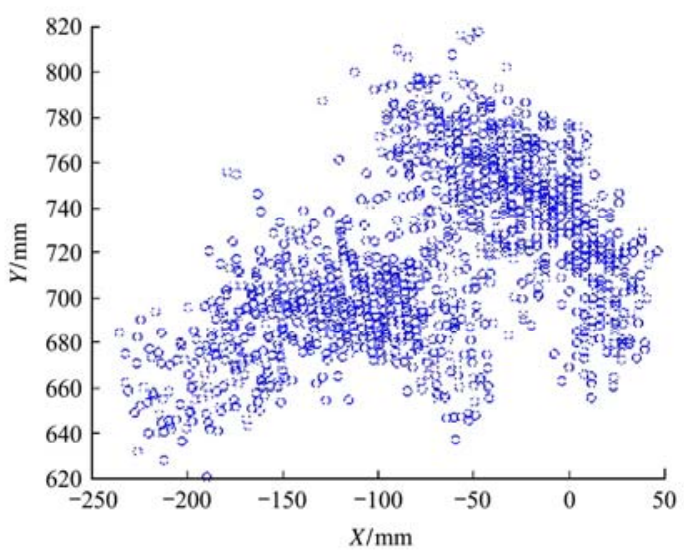

b. Scanning time with $5 \mathrm{~s}$

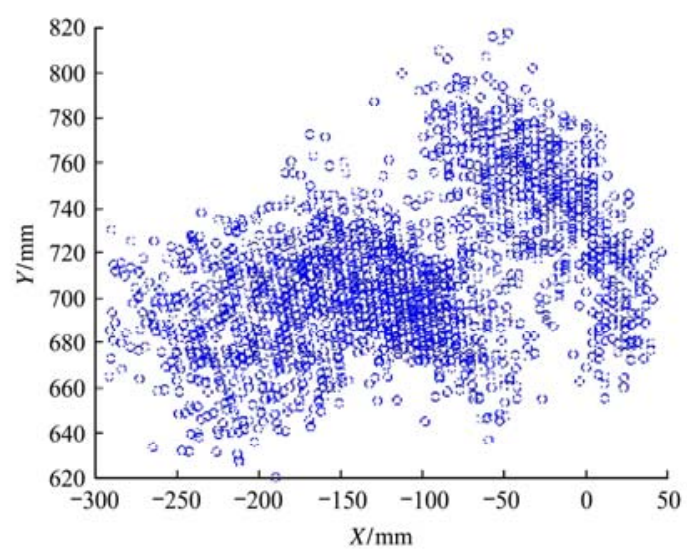

d. Scanning time with $9 \mathrm{~s}$

Figure 7 DD with different scanning time

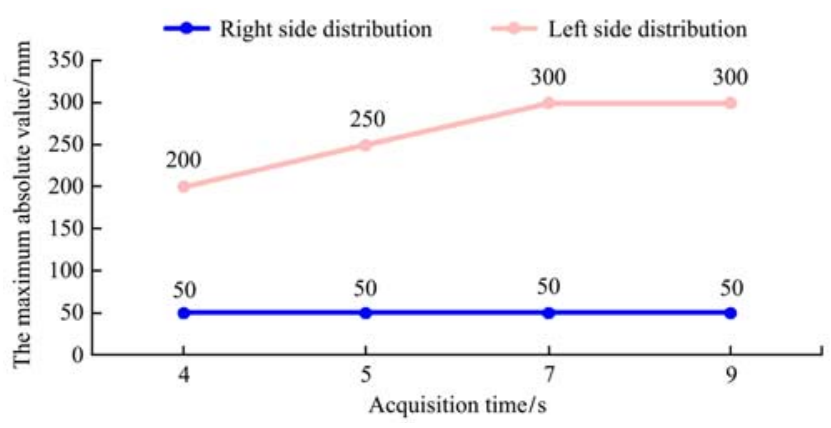

Figure 8 Maximum absolute value of DD on left and right sides

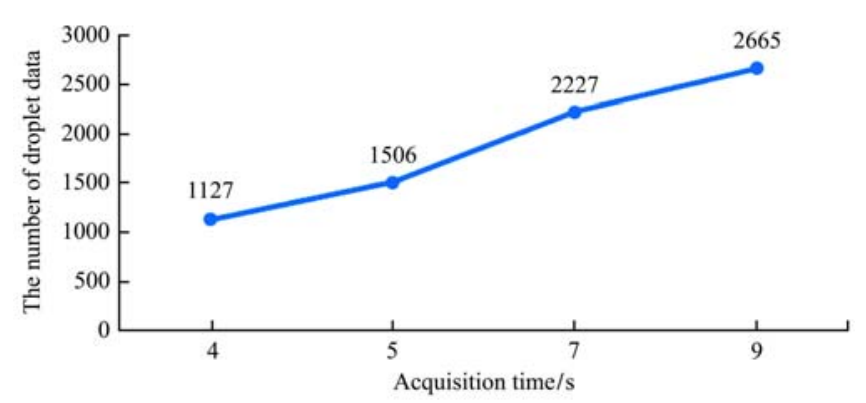

Figure 9 Line chart of droplet sample quantities

2.2.5 Removing of the drift droplets and calculation of the effective range

The "drift droplets" in the paper means that droplets may drift within a small range of two edges, caused by some factors, like the variation of wind speed, the vibration of spraying devices or the disturbance of pressure. This kind of droplets is too few in a unit space to be calculated in the effective range of spray. Therefore, the statistics were used to separate this kind of droplets from the effective range.

For instance, to obtain the range of distribution in transverse $(X$ - direction), the DD is placed in an array first. According to the quantities of data and numeric value, they were divided into several equal intervals, then the probability of these data appearing in different intervals was counted. Furthermore, the frequency histogram of $X$ - direction was drawn. As shown in Figure 10, the distribution of droplets could be examined, combined with the frequency histogram and the curve of normal distribution.

The longitudinal distribution ( $Y$ - direction) is the same. In one direction of distribution, the drifts of droplets 
are much more significant at the edges of two sides in general, so the mean of $X$ array should be calculated by Equation (2).

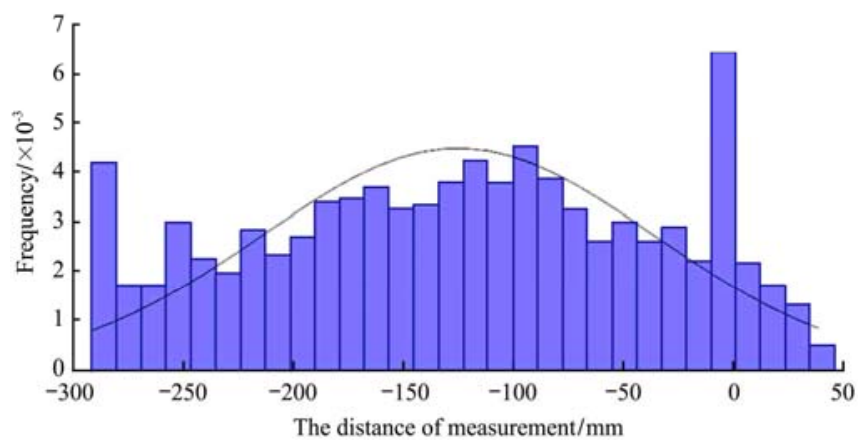

Figure 10 Frequency distribution histogram of DD

$$
\bar{X}=\frac{1}{k} \sum_{i=1}^{k} X_{i}
$$

where, $\bar{X}$ is the average value of $X$ array; $X_{i}$ is the $i^{\text {th }}$ element of $X$ array; $k$ is the quantities of elements of $X$ array.

Taking $\bar{X}$ as a centre, $X$ array is ranked from small to large and divided into two sets.

$$
\begin{aligned}
& X^{\prime}=\operatorname{sort}(X) \\
& X_{L}=\left[X_{1}^{\prime}, X_{2}^{\prime}, \cdots, \bar{X}\right] \\
& X_{R}=\left[\bar{X}, \cdots, X_{k-1}{ }^{\prime}, X_{k}^{\prime}\right]
\end{aligned}
$$

where, $X_{L}$ is the left array; $X_{R}$ is the right array; The function " $\operatorname{sort}()$ " is the sort function; $X_{i}^{\prime}$ is the $i^{\text {th }}$ element of ranked $X$ array.

Afterward, the dynamic proportion $a(a=0.03-0.05$ in general) is set, and the coordinates of the left and right points of segmentation for $X$-axis are computation respectively.

$$
\begin{aligned}
& D_{L}=\bar{X}-\frac{1}{2}(1-\alpha)\left(X_{k}^{\prime}-X_{1}^{\prime}\right) \\
& D_{R}=\bar{X}+\frac{1}{2}(1-\alpha)\left(X_{k}^{\prime}-X_{1}^{\prime}\right)
\end{aligned}
$$

where, $D_{L}$ is the $X$ coordinate of the left point of segmentation; $D_{R}$ is the $X$ coordinate of the right point of segmentation.

Based on these two segmentation points, straight lines that are parallel to the $Y$-axis passing each point are drawn separately, and these are split lines of each side. It could mark off the effective range and drift droplets. Therefore, the effective range of distribution is gained.

$$
D_{e}=D_{R}-D L
$$

where, $D_{e}$ is the effective range of distribution.

Moreover, as is shown in Figure 10, the black curve was the curve of normal distribution and it is marked as $f(x)$ :

$$
f(x)=\frac{1}{\sqrt{2 \pi}} e^{-\frac{\left(x-\mu_{x}\right)^{2}}{2 \sigma_{x}^{2}}}
$$

where, $f(x)$ is the function of normal distribution; $\mu_{x}$ is the mathematical expectation; $\sigma_{x}{ }^{2}$ is the variance. The Equations for $\mu_{x}$ and $\sigma_{x}^{2}$ are listed below:

$$
\mu_{x}=\bar{X}, \sigma_{x}^{2}=\sum_{i=1}^{k}\left(X_{i}^{\prime}-\bar{X}\right)^{2}
$$

According to Equations (6) and (7), it could be judged that whether the distribution of droplets is similar to the normal distribution.

The data of droplets outside any split line are marked by another colour to indicate drift droplets. In Figure 11, two red lines are split lines. The data between the left and right lines are effective droplets and others marked by red colour are drift droplets. The distance from the left line to the right line is the effective range of spray $D_{e}$.

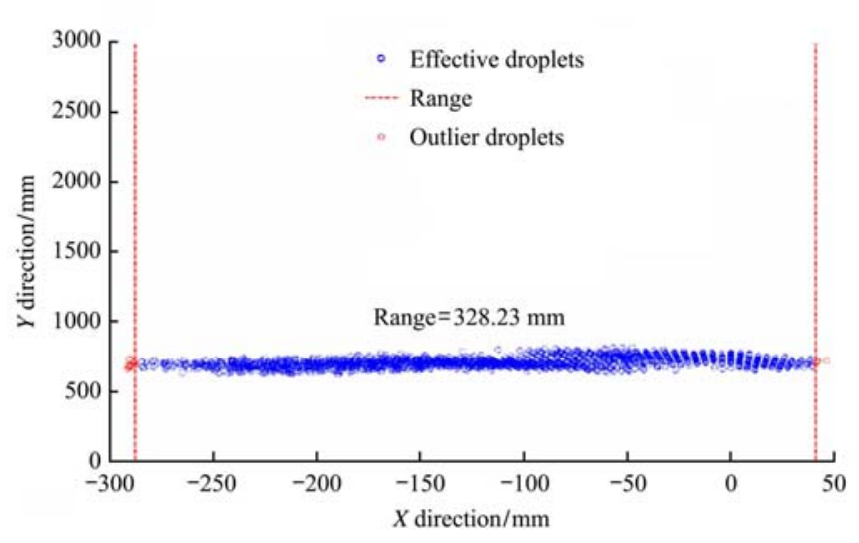

Figure 11 Result of segmentation marker of d DD

2.2.6 Development of 3D outline models of spraying field

The fog field was divided into several sections with equal intervals along the spraying direction, and two LIDARs were set across vertically in each section. As shown in Figure 12, the effective distributions of transverse and longitudinal directions of each section were measured respectively. Multiple equal-interval sections were measured by this way, and the effective ranges of spray were acquired. Meanwhile, the maximum and minimum values are also obtained.

For each section, the maximum and minimum values 
are pointed as coordinate values (Figure 13a). The $Y 1 / X 1$ is the minimum value of $Y / X$ direction and $Y 2 / X 2$ is the maximum value of $Y / X$ direction. And then, segments that are perpendicular to the distribution direction are plotted based on each point. These four segments could form a rectangle to mark the effective range (Figure 13b). At last, the vertexes of one section are linked with other vertexes located in the same position so that the $3 \mathrm{D}$ outline model is developed. The diagram is shown in Figure 13c.

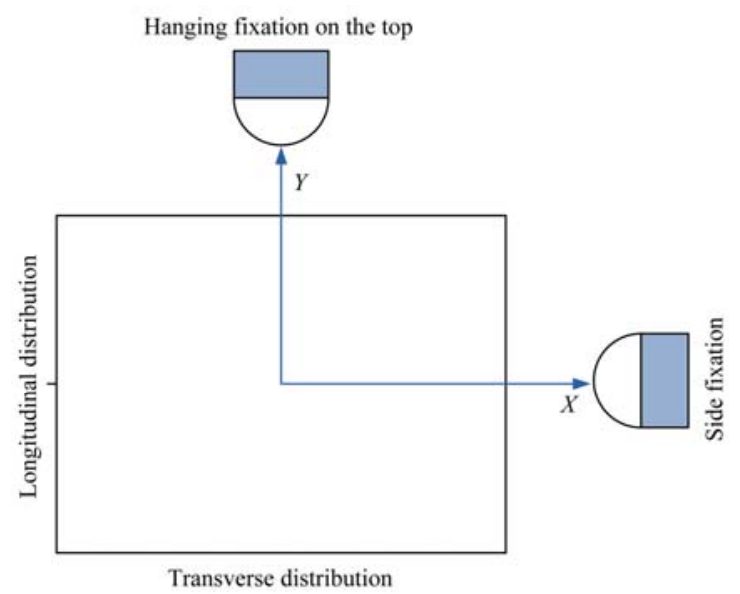

Figure 12 Schematic diagram of cross layout LIDARs in a section

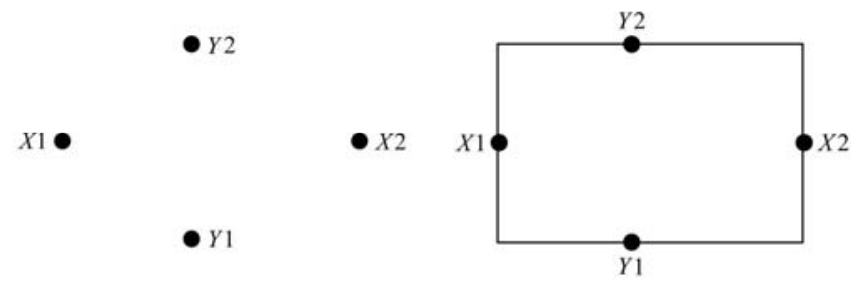

a. Maximum and minimum points $\quad$ b. Rectangle construction

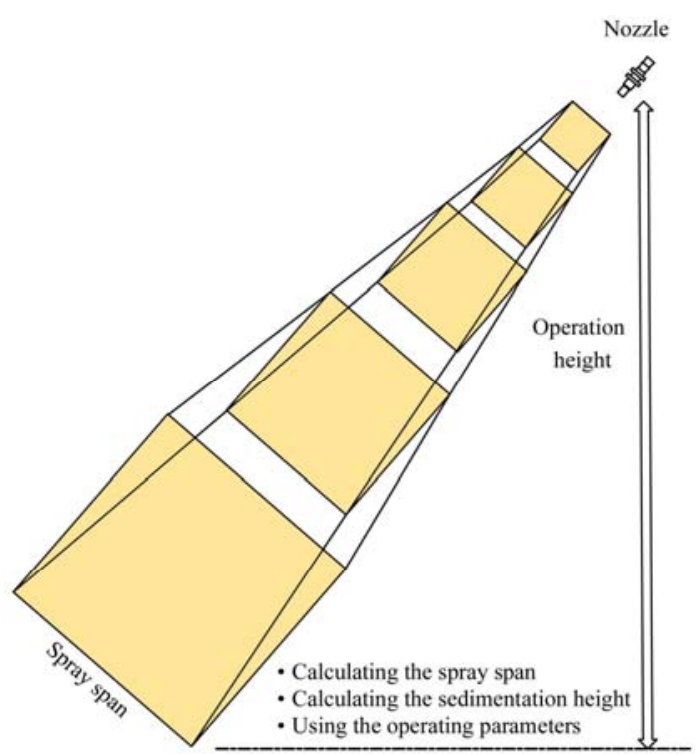

c. 3D outline of fog field

Figure 13 Schematic diagram of droplets 3D-distribution outline

\section{Experiments and analysis}

In order to explore the accuracy and reliability of the study, the verification experiments by an artificial sprayer and the application experiment in the wind tunnel were designed and conducted.

Table 2 Test materials and equipment

\begin{tabular}{cc}
\hline Test materials & Quantities \\
\hline LMS511-20100 LIDAR & 1 \\
PC & 1 \\
Pressure watering can & 1 \\
Regulating pressure nozzle & 1 \\
Wind tunnel & 1 \\
Water sensitive paper & A certain number \\
\hline
\end{tabular}

$P_{0}$ is marked for one pressurizing of the pressure watering can.

\subsection{Verification experiments by an artificial sprayer}

Two kinds of verification experiments by an artificial sprayer were conducted.

\subsubsection{Experiment program}

The tests were carried out in the Lab 453 of College of Engineering in CAU on $19^{\text {th }}$ June 2015.

For the experiment 1, the height from the ground to the spout was set by $88 \mathrm{~cm}$ and the pressure of the artificial sprayer was $15 P_{0}$. Three sections were measured, and the distance between sections and spout were $10 \mathrm{~cm}, 15 \mathrm{~cm}$ and $20 \mathrm{~cm}$. The water absorbent papers were stated in the same position $10 \mathrm{~cm}, 15 \mathrm{~cm}$ and $20 \mathrm{~cm}$ to measure longitudinal ranges of distributions twice separately.

For the experiment 2, the pressure was $15 P_{0}$ and 18 $P_{0}$. The ranges of spray were measured within these two different pressures separately. The height from the ground to the spout was set by $66 \mathrm{~cm}$. The paper was placed on the ground to measure the ranges of distributions of two directions. When the axial distribution was measured, the distance between the artificial sprayer and the LIDAR was $65 \mathrm{~cm}$ in axis direction and $45 \mathrm{~cm}$ in radial direction; it was set by $140 \mathrm{~cm}$ facing the spout when the radial distribution was measured.

In these two experiments, the quality of the artificial sprayer and water was $1000 \mathrm{~g}$ in total.

The results of measurement of LIDAR were marked 
by $Q_{i}(i=1,2, \ldots, n)$, and the mean $P_{1}$ was calculated by Equation (8):

$$
P_{1}=\frac{1}{n} \sum_{i=1}^{n} Q_{i}
$$

The results of water absorbent paper were marked by $P_{2}$, so the relative error $R$ was calculated by Equation (9):

$$
R=1-\frac{P_{2}}{P_{1}} \times 100 \%
$$

The Relative Standard Deviation (RSD) was calculated by Equation (10):

$$
R S D=\frac{\sqrt{\frac{1}{n} \sum_{i=1}^{n}\left(Q_{i}-P_{1}\right)^{2}}}{P_{1}}
$$

The results of $R$ and $R S D$ were used to illustrate the accuracy of the measurement.

\subsubsection{Experiment results}

Some results of these experiments measured by the LIDAR and the water absorbent paper are shown in Figure 14.
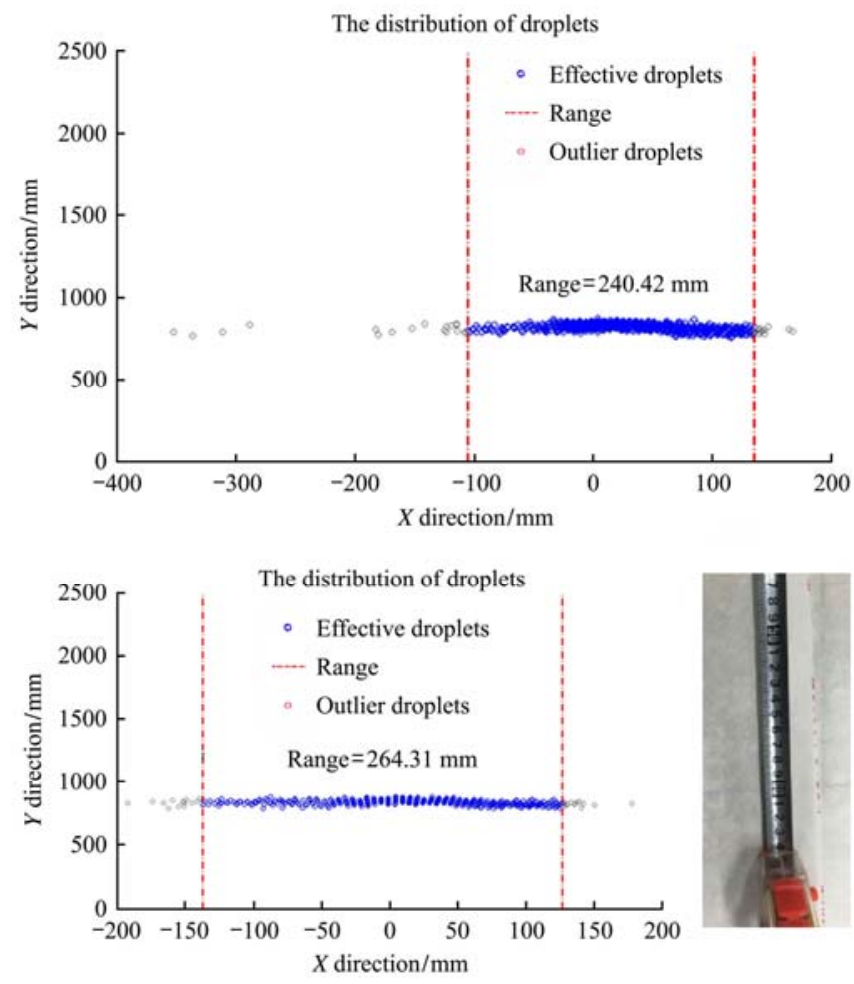

Figure 14 Sample of results

According to the Tables 3 and 4, it is known that in these experiments, compared with the results measured by the water absorbent paper, the relative error by the LIDAR were under $7 \%$ and RSD was less than $16 \%$. It illustrates that this method has a great accuracy and feasibility.
Table 3 Results of experiment 1

\begin{tabular}{|c|c|c|c|c|c|c|}
\hline $\begin{array}{l}\text { Pressure and } \\
\text { direction }\end{array}$ & $\begin{array}{l}\text { Test } \\
\text { No. }\end{array}$ & $Q_{i} / \mathrm{mm}$ & $\begin{array}{c}\text { Standard } \\
\text { deviation } \\
/ \mathrm{mm}\end{array}$ & $\begin{array}{c}P_{1} \\
/ \mathrm{mm}\end{array}$ & $\begin{array}{c}P_{2} \\
\mathrm{~mm}\end{array}$ & $R$ and RSD \\
\hline \multirow{5}{*}{$\begin{array}{l}15 P_{0} \text { axial } \\
\text { distribution }\end{array}$} & 1 & 561.64 & \multirow{5}{*}{65.765} & \multirow{5}{*}{631.310} & \multirow{5}{*}{645} & \multirow{5}{*}{$\begin{array}{c}R=2.13 \% \\
R S D=10.42 \%\end{array}$} \\
\hline & 2 & 639.65 & & & & \\
\hline & 3 & 727.78 & & & & \\
\hline & 4 & 578.72 & & & & \\
\hline & 5 & 648.76 & & & & \\
\hline \multirow{5}{*}{$\begin{array}{l}15 P_{0} \text { radial } \\
\text { distribution }\end{array}$} & 1 & 740.54 & \multirow{5}{*}{141.726} & \multirow{5}{*}{898.626} & \multirow{5}{*}{950} & \multirow{5}{*}{$\begin{array}{c}R=5.41 \% \\
R S D=15.77 \%\end{array}$} \\
\hline & 2 & 1046.29 & & & & \\
\hline & 3 & 1017.01 & & & & \\
\hline & 4 & 761.86 & & & & \\
\hline & 5 & 927.43 & & & & \\
\hline \multirow{5}{*}{$\begin{array}{l}18 P_{0} \text { axial } \\
\text { distribution }\end{array}$} & 1 & 755.86 & \multirow{5}{*}{57.323} & \multirow{5}{*}{752.774} & \multirow{5}{*}{780} & \multirow{5}{*}{$\begin{array}{c}R=3.49 \% \\
R S D=7.61 \%\end{array}$} \\
\hline & 2 & 734.68 & & & & \\
\hline & 3 & 688.56 & & & & \\
\hline & 4 & 845.04 & & & & \\
\hline & 5 & 739.73 & & & & \\
\hline \multirow{5}{*}{$\begin{array}{l}18 P_{0} \text { radial } \\
\text { distribution }\end{array}$} & 1 & 906.32 & \multirow{5}{*}{67.298} & \multirow{5}{*}{929.478} & \multirow{5}{*}{960} & \multirow{5}{*}{$\begin{array}{c}R=3.18 \% \\
R S D=7.24 \%\end{array}$} \\
\hline & 2 & 853.52 & & & & \\
\hline & 3 & 1000.42 & & & & \\
\hline & 4 & 887.02 & & & & \\
\hline & 5 & 1000.01 & & & & \\
\hline
\end{tabular}

\begin{tabular}{ccccc}
\hline Section & $Q_{i} / \mathrm{mm}$ & $P_{1} / \mathrm{mm}$ & $P_{2} / \mathrm{mm}$ & $R$ \\
\hline Section 1 & 240.42 & 252.365 & 250 & $0.94 \%$ \\
& 264.31 & & & \\
Section 2 & 313.30 & 346.600 & 330 & $4.79 \%$ \\
& 379.90 & & & \\
Section 3 & 447.26 & 460.095 & 430 & $6.54 \%$ \\
\hline
\end{tabular}

Table 4 Results of experiment 2

\subsection{Wind tunnel test}

\subsubsection{Test program}

The test was carried out in the Lab of National Precision Agriculture Research and Demonstration Base located in Beijing on $14^{\text {th }}$ July 2016. The main parameters of the wind tunnel are listed in Table 5. The output wind speeds applied in the experiment were $0 \mathrm{~m} / \mathrm{s}$, $5.40 \mathrm{~m} / \mathrm{s}, 12.90 \mathrm{~m} / \mathrm{s}$.

Table 5 Main parameters of the wind tunnel

\begin{tabular}{cc}
\hline Parameters & Specification \\
\hline Form of structure & open-circuit closed wind tunnel \\
Diameter of test section & $600 \mathrm{~mm}(\mathrm{H}) \times 600 \mathrm{~mm}(\mathrm{~W})$ \\
Flow velocity & $0-46 \mathrm{~m} / \mathrm{s}$ \\
Turbulence intensity & $<0.3 \%$ \\
Flow uniformity & $<0.5 \%$ \\
Dynamic pressure stability coefficients & $<1.0 \%$ \\
Averaged flow inclination angle & $<1.0^{\circ}$ \\
Normalized axial static pressure gradient & $<0.02$ \\
\hline
\end{tabular}


The fog field was divided into three sections, and the transverse and longitudinal distributions of each section were measured with these three kinds of wind speeds. Furthermore, the 3D outline models of each condition were developed. The photo of the test site is shown in Figure 15, in which the LIDAR is circled by the yellow frame.

\subsubsection{Test results}

According to the Table 6, in the same wind speed, the farther distance between the nozzle and the section, the longer ranges of transverse and longitudinal distributions. However, within the different wind speeds, there was no regularity for the section located in the same position. The results of 3D outline models are shown in Figure 16.

According to the Figure 16 and Table 6, the fog field had the same trend of downward deposition. Moreover, it is known that the models are clear and intuitive and the deposition of the fog field could be examined. Therefore, it illustrates that the method of development of the $3 \mathrm{D}$ outline model has practicability.

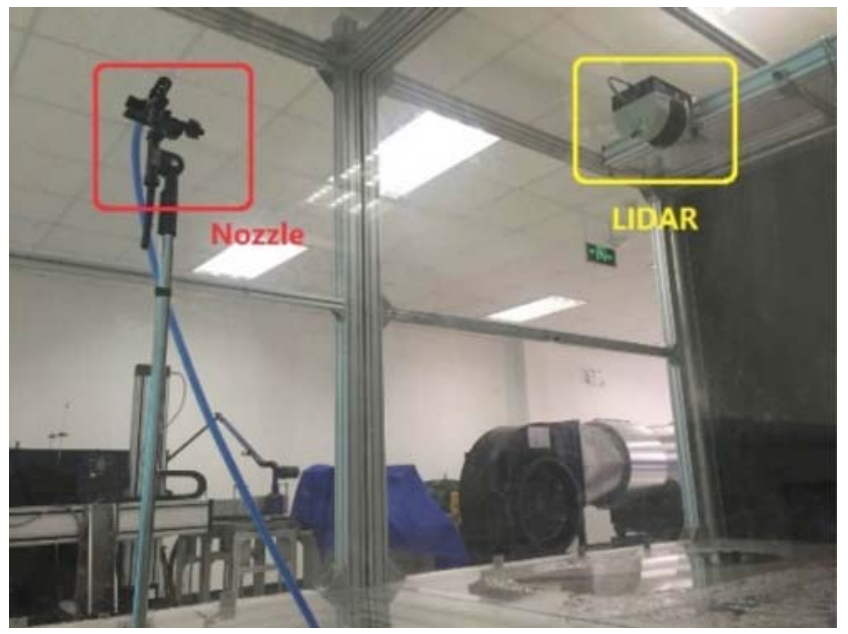

Figure 15 Photo of the test site

Table 6 Results of three sections with three wind speeds

\begin{tabular}{|c|c|c|c|c|c|c|c|c|c|}
\hline \multirow{2}{*}{$\begin{array}{l}\text { Wind } \\
/ \mathrm{m} \cdot \mathrm{s}^{-1}\end{array}$} & \multicolumn{3}{|c|}{ Section 1} & \multicolumn{3}{|c|}{ Section2 } & \multicolumn{3}{|c|}{ Section 3} \\
\hline & $\begin{array}{c}\mathrm{X} \\
/ \mathrm{mm}\end{array}$ & $\begin{array}{c}\mathrm{Z} \\
/ \mathrm{mm}\end{array}$ & $\begin{array}{l}\text { Area } \\
/ \mathrm{mm}^{2}\end{array}$ & $\begin{array}{c}\mathrm{X} \\
/ \mathrm{mm}\end{array}$ & $\begin{array}{c}\mathrm{Z} \\
/ \mathrm{mm}\end{array}$ & $\begin{array}{l}\text { Area } \\
/ \mathrm{mm}^{2}\end{array}$ & $\begin{array}{c}\mathrm{X} \\
/ \mathrm{mm}\end{array}$ & $\begin{array}{c}\mathrm{Z} \\
/ \mathrm{mm}\end{array}$ & $\begin{array}{l}\text { Area } \\
/ \mathrm{mm}^{2}\end{array}$ \\
\hline 0 & 458.99 & 111.57 & 51209.51 & 630.41 & 183.87 & 115913.49 & 743.78 & 146.67 & 109090.21 \\
\hline 5.4 & 458.99 & 91.24 & 41878.25 & 615.21 & 140.55 & 86467.77 & 705.07 & 128.23 & 90411.13 \\
\hline 12.9 & 417.74 & 86.27 & 36038.43 & 652.53 & 140.32 & 91563.01 & 656.68 & 185.48 & 21801.01 \\
\hline
\end{tabular}

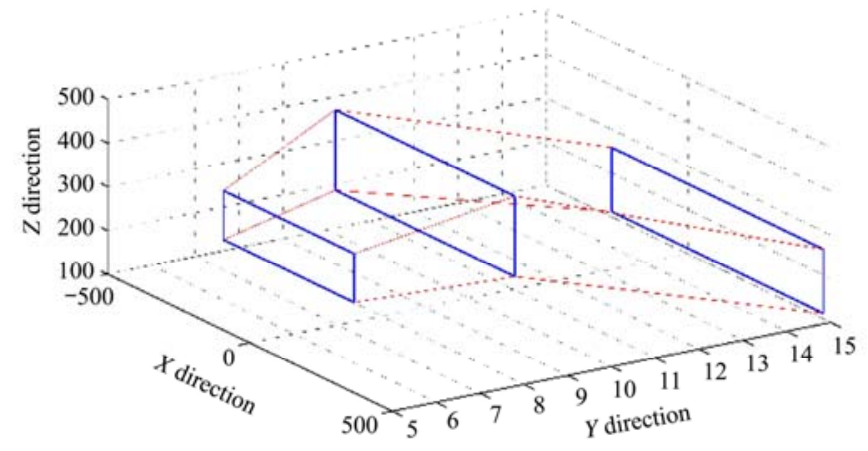

a. 3D outline model of $0 \mathrm{~m} / \mathrm{s}$

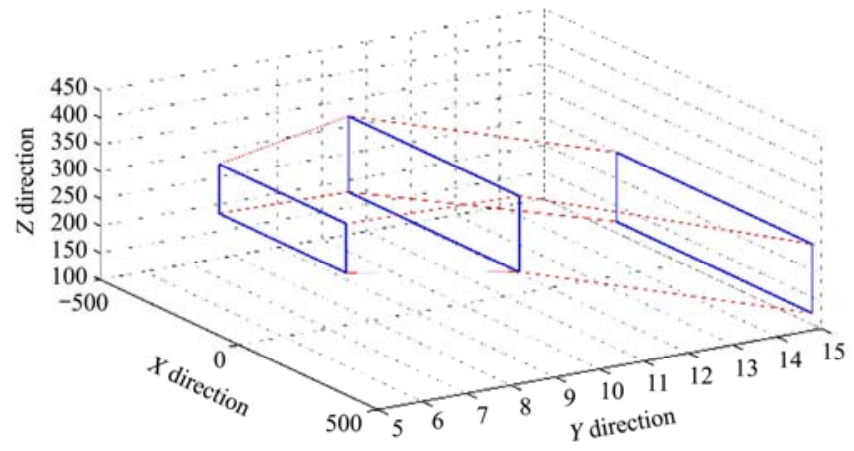

b. 3D outline model of $5.40 \mathrm{~m} / \mathrm{s}$

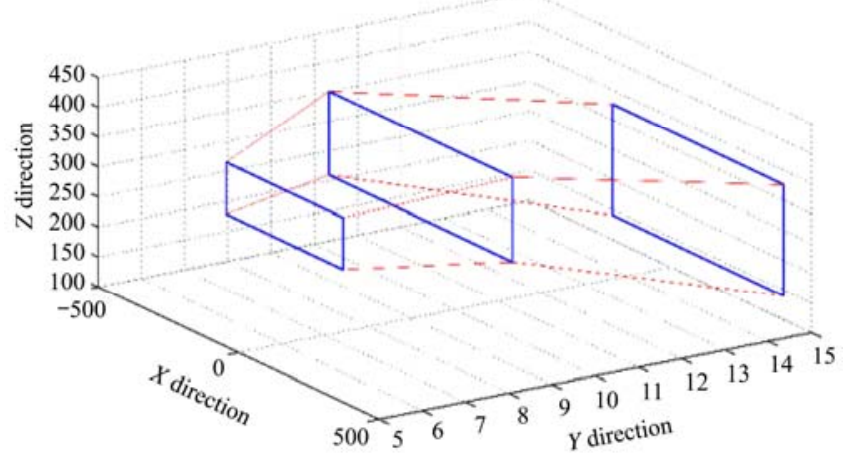

c. 3D outline model of $12.90 \mathrm{~m} / \mathrm{s}$

Figure 16 3D outline model of different wind speeds 


\section{Conclusions}

This research was aimed at the key point of the detection and measurement of droplet distribution for spray operation in agricultural aviation, and the method of measurement of the distribution of droplets based on LIDARs was proposed. The algorithms of the recognition and extraction of targets, the calculation of the range of distribution and development of 3D distribution model were studied, in which the droplets were as the primary object. In addition, the tests imitating the operation condition were carried out, so that the accuracy and practicability of the method were investigated. As a consequence, the conclusion is summarized below:

(1) The designed method was based on the LIDAR-LMS511-20100. Combined with hardware devices and droplets/fog field description methods, the droplets or fog field distribution could be obtained quickly and intuitively. Compared with the artificial detection, the relative error was under 7\% and RSD was under $16 \%$. The results were intuitive.

(2) The results of the various experiments showed that this method had the advantages, such as short period of scanning and high precision. The detection system using this method could be stable when operating in practical environment and conditions. Thus, it would be applicable for measuring the spraying range and evaluating nozzles as well as majority experiment conditions, such as the tests in wind tunnel and measurement of spray range of UAVs for plant protection before operating.

\section{Acknowledgements}

This research was financially supported by the National Key Research and Development Program of China (No. 2016YFD0200700) from China Ministry of Science and Technology, and the Fundamental Research Funds for the Central Universities (No. 2015TC036).

\section{[References]}

[1] Qin W C, Xue X Y, Zhou L X, Zhang S C, Sun Z, Kong W, et al. Effects of spraying parameters of unmanned aerial vehicle on droplets deposition distribution of maize canopies. Transactions of the CSAE, 2014; 30(5): 50-56. (in Chinese )

[2] Zhang P, Deng L, Lyu Q, He S L, Yi S L, Liu Y D, et al. Effects of citrus tree-shape and spraying height of small unmanned aerial vehicle on droplet distribution. Int J Agric \& Biol Eng, 2016; 9(4): 45-52.

[3] Ru Y, Jin L, Zhou H P, Jia Z C. Charging characteristics of droplets effect on the distribution and adhesion of droplets on target. Journal of Nanjing Forestry University (Natural Sciences Edition), 2014; 38(3): 129-133. (in Chinese)

[4] Song S R, Hong T S, Wang W X, Zhang H X, Luo X W, Francis S. Testing analysis on deposit and distribution of pesticide spraying in rice fields. Transactions of the CSAM, 2004; 35(6): 90 - 93. (in Chinese )

[5] Zheng Y J, Yang S H, Zhao C J, Chen L P, Lan Y B, Tan Y. Modelling operation parameters of UAV on spray effects at different growth stages of corns. Int J Agric \& Biol Eng, 2017; 10(3): 57-66.

[6] Qin W C, Xue X Y, Cui L F, Zhou Q Q, Xu Z F, Chang F L. Optimization and test for spraying parameters of cotton defoliant sprayer. Int J Agric \& Biol Eng, 2016; 9(4): 63-72.

[7] Bonds J A, Mulla C, Hunter E. Characterization of the effects of droplet size, air blast strength, and angle on spray distribution and efficacy of a buffalo turbine barrier treatment sprayer. Journal of ASTM International, 2011; 8(5): 1-6.

[8] Wolf R E. Comparing downwind spray droplet deposits of four flat-fan nozzle types measured in a wind tunnel and analyzed using dropletscan software. Applied Engineering in Agriculture, 2005; 21(2): 173-177.

[9] De Boer D W, Monnens M J, Kincaid D C. Measurement of sprinkler droplet size. Transactions of the ASABE, 2001; 17(1): 11-15.

[10] Guan X P. Effects of Operating Parameters for Unmanned Aerial Vehicles on Spraying Deposition. Hubei Agricultural Sciences, 2014; 53(3): 678-680. (in Chinese).

[11] Qiu B J, Wang L W, Cai D L, Wu J H, Ding G R, Guan X P. Effects of flight altitude and speed of unmanned helicopter on spray deposition uniform. Transactions of the CSAE, 2013; 29(24): 25-32. (in Chinese)

[12] Foqué D, Nuyttens D. Effect of air support and spray angle on coarse droplet sprays in ivy pot plants. Transactions of the ASABE, 2011; 54(2): 409-416.

[13] Qi L J, Hu K Q, Mang L, Wang H T. Droplet detection based on image processing. Transactions of the CSAM, 2009; 40(Supp.1): 48-51. (in Chinese)

[14] Lu J, Li P P, Qiu B J, Jia W D. Analysis of charged droplet characteristics and deposition motion in agricultural high voltage electrostatic spraying field. High Voltage Engineering, 2009; 35(5): 1077-1082. (in Chinese)

[15] Salyani M, Fox R D. Performance of image analysis for 
assessment of simulated spray droplet distribution. Transactions of the ASAE, 1994; 37(4): 1083-1089.

[16] Zhang H C, Dorr Gary, Zheng J Q, Zhou H P. Wind tunnel experiment of influence on droplet size distribution of flat fan nozzles. Transactions of the CSAM, 2012; 43(6): 53-57. (in Chinese)

[17] Wedding J B, Kim Y J. Wind tunnel characterization of aerial spray nozzles using the laser particle spectral analyzer. Optical Engineering, 1986; 25(4): 556-560.

[18] Sidahmed M M, Yates W E. Measuring spray droplets with PMS-FSSP probes. Transactions of the ASAE, 1997; 40(5): 1237-1242.

[19] Endalew A M, Debaer C, Rutten N, Vercammen J, Delele M $\mathrm{A}$, Ramon $\mathrm{H}$, et al. A new integrated CFD modelling approach towards air-assisted orchard spraying. Part I. Model development and effect of wind speed and direction on sprayer airflow. Computers and Electronics in Agriculture, 2010; 71(2): 128-136.

[20] Crowe T G, Downey D, Giles D K. Digital device and technique for sensing distribution of spray deposition. Transactions of the ASAE, 2005; 48(6): 2085-2093.

[21] Zheng Y J, Lan Y B, Kang F, Ma C, Chen H, Tan Y. Using laser sensor for measuring crop conditions in precision agriculture. 2013 ASABE Annual International Meeting, 2013: 2595-2602.

[22] Shao Q, Xu T, Yoshino T, Zhao Y, Yang W, Zhu H. Point cloud online measurement of stored bulk grain. Int J Agric \& Biol Eng, 2016; 9(1): 71-78.

[23] Zhao T, Noguchi N, Yang L L, Ishii K, Chen J. Development of uncut crop edge detection system based on laser rangefinder for combine harvesters. Int J Agric \& Biol Eng, 2016; 9(2): 21-28.

[24] Wang Y X, Xu S S, Li W B, Kang F, Zheng Y J. Identification and location of grapevine sucker based on information fusion of 2D laser scanner and machine vision. Int J Agric \& Biol Eng, 2017; 10(2): 84-93.

[25] Zhang Y Y. Zhou J. Laser Radar Based Orchard Trunk Detection. Journal of China Agricultural University, 2015; 20(5): 249-255. (in Chinese)

[26] Xue J L, Zhang S S. Navigation of an agricultural robot based on laser radar. Transactions of the CSAM, 2014; 45(9): 55-60. (in Chinese)

[27] SICK Company. The laser scanning and measurement system: Area scan and profile measurement. 1-62. (in Chinese) 